\title{
Parametrization of the Hybrid Potential for Pairs of Neutral Atoms
}

\author{
Kevin Cahil:* \\ Department of Physics and Astronomy, University of New Mexico, Albuquerque, NM 87131
}

(Dated: October 27, 2018)

\begin{abstract}
The hybrid form is a combination of the Rydberg potential and the London inverse-sixth-power energy. It is accurate at all relevant distance scales and simple enough for use in all-atom simulations of biomolecules. One may compute the parameters of the hybrid potential for the ground state of a pair of neutral atoms from their internuclear separation, the depth and curvature of their potential at its minimum, and from their van der Waals coefficient of dispersion $C_{6}$.
\end{abstract}

PACS numbers: 34.20.Cf (Interatomic potentials and forces), 87.15.-v, 82.35.Pq, 77.84.Jd

\section{THE HYBRID FORM}

Commercial molecular-modeling codes use pair potentials chosen more for speed than for accuracy. They use the harmonic potential

$$
V_{h}(r)=-E_{0}+\frac{k}{2}\left(r-r_{0}\right)^{2}
$$

for covalently bonded pairs of neutral atoms and the Lennard-Jones potential [1]

$$
V_{L J}(r)=E_{0}\left[\left(\frac{r_{0}}{r}\right)^{12}-2\left(\frac{r_{0}}{r}\right)^{6}\right]
$$

for unbonded pairs. The recently introduced hybrid potential [2]

$$
V(r)=a e^{-b r}(1-c r)-\frac{C_{6}}{r^{6}+d r^{-6}}
$$

is nearly as fast and much more accurate. It is fast enough for use in all-atom simulations of biomolecules and accurate at all biologically relevant distance scales when its parameters are suitably chosen. How to choose them is the focus of this paper. Formulas are derived for $a, b, c, \& d$ in terms of the internuclear separation $r_{0}$, the depth $E_{0}$ and curvature $k$ of the potential at its minimum, and the van der Waals coefficient $C_{6}$ of the pair. The hybrid potential therefore is applicable to pairs of neutral atoms for which no empirical potential is available. Its eventual incorporation into TINKER [3], Amber [4], and other such codes is a goal of this work.

The hybrid potential is a combination of the Rydberg formula used in spectroscopy and the London formula for pairs of atoms. The terms involving $a, b$, and $c$ were proposed by Rydberg to incorporate spectroscopic data, but were largely ignored until recently. The constant $C_{6}$ is the coefficient of the London tail. The new term $d r^{-6}$ cures the London singularity. As $r \rightarrow 0, V(r) \rightarrow a$, finite; as $r \rightarrow \infty, V(r)$ approaches the London term, $V(r) \rightarrow-C_{6} / r^{6}$. In a perturbative analysis [5], the

*Electronic address: cahill@unm.edu $a, b, c$ terms arise in first order, and the $C_{6}$ term in second order.

When fitted to spectroscopically determined potentials for the ground states of $\mathrm{H}_{2}, \mathrm{~N}_{2}, \mathrm{O}_{2}, \mathrm{Ar}-\mathrm{Ar}$, and $\mathrm{Kr}-\mathrm{Kr}$, the hybrid form is four orders of magnitude more accurate than the harmonic and Lennard-Jones potentials and five times more accurate than the Morse 6], Varnshi [7], and Hulburt-Hirschfelder [8] potentials [2]. It also yields accurate second virial coefficients and heats of vaporization [2]. Its simplicity recommends it as a teaching tool and as a practical form for computation.

How does one find the parameters $a, b, c, \& d$ when an empirical potential is not available? For many pairs of neutral atoms, spectroscopists have measured the internuclear separation $r_{0}$, the well depth $E_{0}=\left|V\left(r_{0}\right)\right|$ and curvature $k=V^{\prime \prime}\left(r_{0}\right)$ at the minimum of the potential, and the London coefficient $C_{6}$. These input parameters are discussed in Sec. II with an emphasis on the curvature $k$ and its relation to the vibrational frequency of the ground state and to the energy $D_{0}$ needed to dissociate the ground state. Section III develops a rule of thumb for the parameter $d$ to which the hybrid form is relatively insensitive. Section IV derives formulas for the hybrid parameters $a, b, \& c$ in terms of $r_{0}, E_{0}, k, C_{6}, \& d$ and plots the resulting hybrid potentials for the 11 pairs $\mathrm{H}_{2}, \mathrm{~N}_{2}, \mathrm{O}_{2}, \mathrm{NO}, \mathrm{OH}, \mathrm{I}_{2}, \mathrm{Li}_{2}, \mathrm{Na}_{2}, \mathrm{~K}_{2}, \mathrm{Ar}-\mathrm{Ar}$, \& $\mathrm{Kr}-\mathrm{Kr}$.

\section{INPUT PARAMETERS}

In addition to the well depth $E_{0}=\left|V\left(r_{0}\right)\right|$, the internuclear separation $r_{0}$ at the minimum, and the London or van der Waals coefficient $C_{6}$, the curvature $k=V^{\prime \prime}\left(r_{0}\right)$ of the potential at $r_{0}$ often is available either directly or in the guise of the energy of dissociation $D_{0}$.

Near $r_{0}$, the motion of the internuclear separation $r$ is described by the lagrangian

$$
L=\frac{1}{2} \mu \dot{r}^{2}-\frac{1}{2} k\left(r-r_{0}\right)^{2}
$$

in which $\mu=m_{1} m_{2} /\left(m_{1}+m_{2}\right)$ is the reduced mass of the two atoms of mass $m_{1}$ and $m_{2}$. To lowest order in $r-r_{0}$, the ground state then has energy

$$
E_{g}=\frac{1}{2} \hbar \omega-E_{0}
$$


in which $\omega$ is the (angular) vibrational frequency of the ground state. The curvature $k$ is related to $\omega$ by

$$
k=\mu \omega^{2} .
$$

In the chemical-physics literature, the depth $E_{0}$ and separation $r_{0}$ are labeled $D_{e}$ and $r_{e}$, and the angular frequency $\omega=2 \pi \nu$ is expressed as a frequency $\omega_{e}$ in inverse centimeters: $\omega_{e}=\omega /(2 \pi c)$ where $c$ is the speed of light. In these terms and in cgs units, $k=4 \pi^{2} c^{2} \mu \omega_{e}^{2}$. The energy of dissociation is $D_{0}=-E_{g}=E_{0}-\hbar \omega / 2$, and so the curvature $k=V^{\prime \prime}\left(r_{0}\right)$ is related to the difference between it and the well depth $E_{0}=D_{e}$ by

$$
k=4 \mu\left(E_{0}-D_{0}\right)^{2} / \hbar^{2}=4 \mu\left(D_{e}-D_{0}\right)^{2} / \hbar^{2} .
$$

The values of the input parameters are listed in Table I. The internuclear separation $r_{0}$ at the minimum of the potential well $V(r)$ and its depth $E_{0}=V\left(r_{0}\right)$ are from [9] for $\mathrm{H}_{2}, \mathrm{NO}, \mathrm{OH}, \& \mathrm{I}_{2}$, and from [10] for $\mathrm{O}_{2}$. The curvature $k=V^{\prime \prime}\left(r_{0}\right)$ of the potential at its minimum is from [9] for $\mathrm{H}_{2}, \mathrm{O}_{2}, \mathrm{NO}, \mathrm{OH}, \& \mathrm{I}_{2}$. The van der Waals coefficient $C_{6}$ is from 11] for $\mathrm{H}_{2}$; from [12] for $\mathrm{NO}$; from [13] for $\mathrm{OH}$; and from [14] for $\mathrm{O}_{2}, \mathrm{I}_{2}, \mathrm{Ar}-\mathrm{Ar}$, \& $\mathrm{Kr}-\mathrm{Kr}$. The values of $r_{0}, E_{0}, \& k$ for $\mathrm{Ar}-\mathrm{Ar}$ and $\mathrm{Kr}-\mathrm{Kr}$ respectively are from [15] and [16]. The values of $r_{0}, E_{0}$, $k, \& C_{6}$ for $\mathrm{N}_{2}, \mathrm{Li}_{2}, \mathrm{Na}_{2}, \& \mathrm{~K}_{2}$ respectively are from [17]; [18]; [14], 19], \& 20]; and [14], 20], 21], \& [22].

\section{A RULE OF THUMB}

The $d$-term of the hybrid form is a trick to avoid the $1 / r^{6}$ singularity of the London term. It has no other justification. Luckily, the quality of the fit is not sensitive to the precise value of the parameter $d$, and so we need only a good rule of thumb for that parameter. To develop an approximate formula that estimates this parameter, we first find values of $a, b, c, \& d$ that provide good fits of the hybrid form $V(r)$ to empirical potentials for the 11 pairs $\mathrm{H}_{2}, \mathrm{~N}_{2}, \mathrm{O}_{2}, \mathrm{NO}, \mathrm{OH}, \mathrm{I}_{2}, \mathrm{Li}_{2}, \mathrm{Na}_{2}, \mathrm{~K}_{2}, \mathrm{Ar}-\mathrm{Ar}$, \& $\mathrm{Kr}-\mathrm{Kr}$ of neutral atoms.

Empirical potentials obtained from spectroscopic data [10, 23, 24] by the RKR (Rydberg 25], Klein [26], Rees [27]) method are available from [9] for $\mathrm{H}_{2}, \mathrm{NO}, \&$ $\mathrm{I}_{2}$; from [28] for $\mathrm{OH}$; from [17] for $\mathrm{N}_{2}$; from 10] for $\mathrm{O}_{2}$; from [18] for $\mathrm{Li}_{2}$; from [19] for $\mathrm{Na}_{2}$; from [29] for $\mathrm{K}_{2}$; from [15] for $\mathrm{Ar}-\mathrm{Ar}$; and from [16] for $\mathrm{Kr}-\mathrm{Kr}$.

Section IV] contains formulas [15, 17, 19, \& 20) for $a, b, \& c$ in terms of $E_{0}, r_{0}, k, C_{6}, \& d$ that ensure that the hybrid form $V(r)$ goes through the minimum $\left(r_{0},-E_{0}\right)$ with curvature $k$. For each pair of atoms, I found the value of $d$ that best fits the hybrid form $V(r)$ to the empirical potential of the pair for $r \geq f r_{0}$ in which $0 \leq f \leq 1$. The resulting values of $a, b, c, \& d$ are listed in Table $\amalg$ along with the fractions $f$ and the root-mean-square (rms) errors $\Delta V(r)$ for $r \geq f r_{0}$. Figures 111] show that the fitted hybrid forms $V_{f}$ (dashes, dark blue) nicely follow the points (diamonds, cyan) of
TABLE I: The depth $E_{0}=V\left(r_{0}\right)$ of the potential well $V(r)$, the internuclear separation $r_{0}$ and the curvature $k=V^{\prime \prime}\left(r_{0}\right)$ at the minimum $r=r_{0}$, and the van der Waals coefficient $C_{6}$ for 11 pairs of neutral atoms.

\begin{tabular}{|c|c|c|c|c|}
\hline \hline & $E_{0}(\mathrm{eV})$ & $r_{0}(\AA)$ & $k\left(\mathrm{eV} \AA^{-2}\right)$ & $C_{6}\left(\mathrm{eV}^{6}\right)$ \\
\hline $\mathrm{H}_{2}$ & 4.7467 & 0.7417 & 35.8861 & 3.88338 \\
\hline${ }^{14} \mathrm{~N}_{2}$ & 9.8995 & $1.09768^{a}$ & 143.2245 & 14.382 \\
\hline $\mathrm{O}_{2}$ & 5.2136 & 1.2075 & 73.4726 & 9.3215 \\
\hline $\mathrm{NO}$ & 6.609 & 1.1590 & 99.5676 & 11.245 \\
\hline $\mathrm{OH}$ & 4.624 & 0.9707 & 48.6196 & 6.854 \\
\hline $\mathrm{I}_{2}$ & 1.5571 & 2.668 & 10.7378 & 230.05 \\
\hline $\mathrm{Li}_{2}$ & 1.0559 & 2.6730 & 1.5752 & 829.33 \\
\hline $\mathrm{Na}_{2}$ & 0.74664 & 3.0786 & 1.0706 & 929.76 \\
\hline $\mathrm{K}_{2}$ & 0.55183 & 3.9243 & 0.61375 & 2328.6 \\
\hline $\mathrm{Ar}_{2}$ & 0.01234 & 3.757 & 0.0691 & 38.4213 \\
\hline $\mathrm{Kr}_{2}$ & 0.01735 & 4.017 & 0.0896 & 77.6791 \\
\hline \hline
\end{tabular}

${ }^{a}$ Extra digits are included to avoid round-off errors.

TABLE II: The values of the coefficients $a, b, c, \& d$ that fit the hybrid potential $V(r)$ (Eq. (3) ) to RKR data for $\mathrm{H}_{2}, \mathrm{~N}_{2}$, $\mathrm{O}_{2}, \mathrm{NO}, \mathrm{OH}, \mathrm{I}_{2}, \mathrm{Li}_{2}, \mathrm{Na}_{2}, \& \mathrm{~K}_{2}$ and to empirical potentials for $\mathrm{Ar}-\mathrm{Ar} \& \mathrm{Kr}-\mathrm{Kr}$ while respecting Eqs. (15, 17,19 \& 20). The rms errors $\Delta V(r)$ for $r \geq f r_{0}$ are also listed.

\begin{tabular}{|c|c|c|c|c|c|}
\hline \hline & $a(\mathrm{eV})$ & $b\left(\AA^{-1}\right)$ & $c\left(\AA^{-1}\right)$ & $d\left(\AA^{12}\right)$ & $f, \Delta V(\mathrm{eV})$ \\
\hline $\mathrm{H}_{2}$ & 47.796 & 2.9632 & 2.5406 & 12.2 & $0.68,0.086$ \\
\hline $\mathrm{N}_{2}$ & 3752.644 & 4.3533 & 1.1777 & 34.8 & $0.81,0.088$ \\
\hline $\mathrm{O}_{2}$ & 2901.580 & 4.2173 & 1.0510 & 59.8 & $0.84,0.039$ \\
\hline $\mathrm{NO}$ & 3809.497 & 4.4196 & 1.0943 & 47.0 & $0.81,0.077$ \\
\hline $\mathrm{OH}$ & 377.804 & 3.6909 & 1.4668 & 32.5 & $0.80,0.023$ \\
\hline $\mathrm{I}_{2}$ & 14361.15 & 2.8013 & 0.4351 & $2.08 \mathrm{e} 5^{b}$ & $0.88,0.039$ \\
\hline $\mathrm{Li}_{2}$ & 199.481 & 1.6200 & 0.5101 & $2.85 \mathrm{e} 6$ & $0.86,0.016$ \\
\hline $\mathrm{Na}_{2}$ & 231.900 & 1.5311 & 0.4292 & $9.40 \mathrm{e} 6$ & $0.81,0.009$ \\
\hline $\mathrm{K}_{2}$ & 325.051 & 1.3409 & 0.3269 & $9.94 \mathrm{e} 7$ & $0.85,0.008$ \\
\hline $\mathrm{Ar}_{2}$ & 1987.943 & 2.6517 & 0.2978 & $8.10 \mathrm{e} 7$ & $0.70,0.00009$ \\
\hline $\mathrm{Kr}_{2}$ & 1875.462 & 2.3661 & 0.2789 & $5.72 \mathrm{e} 8$ & $0.90,0.00018$ \\
\hline \hline
\end{tabular}

${ }^{b_{2} .083 \mathrm{e} 5}=2.083 \times 10^{5}$.

the empirical potentials for the 11 pairs of neutral atoms $\mathrm{H}_{2}, \mathrm{~N}_{2}, \mathrm{O}_{2}, \mathrm{NO}, \mathrm{OH}, \mathrm{I}_{2}, \mathrm{Li}_{2}, \mathrm{Na}_{2}, \mathrm{~K}_{2}, \mathrm{Ar}-\mathrm{Ar}$, \& $\mathrm{Kr}-$ $\mathrm{Kr}$. The fitted $V_{f}(r)$ 's go through the empirical minima with the right curvatures and closely trace the empirical potentials, at least for $r \geq f r_{0}$. Figure 1 for molecular hydrogen adds the harmonic potential $V_{h}$ (dot-dash, magenta), which fits only near the minimum at $r=r_{0}$. Figure 2 for molecular nitrogen and Fig. 10] for a pair of argon atoms include the Lennard-Jones potential $V_{L J}$ (dot-dash, magenta), which is accurate only near $r_{0}$.

The fitted values of the parameter $d$ in Table II are less well defined than those of $a, b$, and $c$. The empirical rule of thumb

$$
d=7.1 \AA^{12}+2.89 \frac{C_{6}^{3}}{E_{0}^{3} r_{0}^{6}}+0.468 \frac{E_{0}}{\mathrm{eV}} \frac{r_{0}^{13}}{\AA}
$$

gives the 11 values of $d$ listed in Table III and roughly approximates those of Table II 


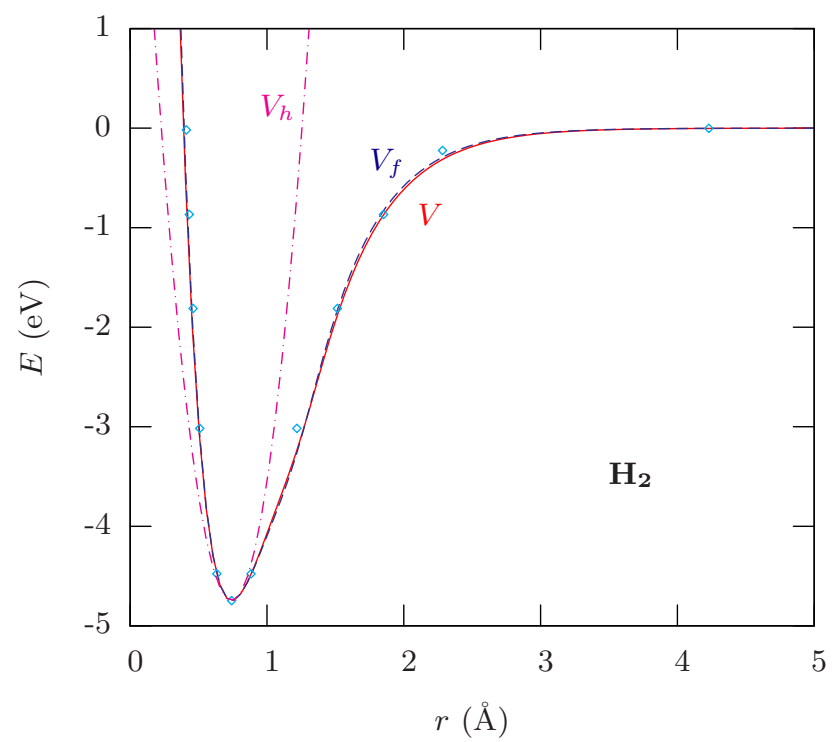

FIG. 1: The hybrid form $V$ with the calculated coefficients $(a$, $b, c, \& d$, Table III) (solid, red) fits the RKR spectral points for the ground state of molecular hydrogen (diamonds, cyan) and gives the correct London tail for $r>3 \AA$ nearly as well as does the hybrid form $V_{f}$ with the fitted coefficients $(a, b, c, \&$ $d$, Table III) (dashes, dark blue). In all the figures, the values of $C_{6}$ used in $V$ and $V_{f}$ are from Table I. The harmonic form $V_{h}$ (dot-dash, magenta) fits only near the minimum.

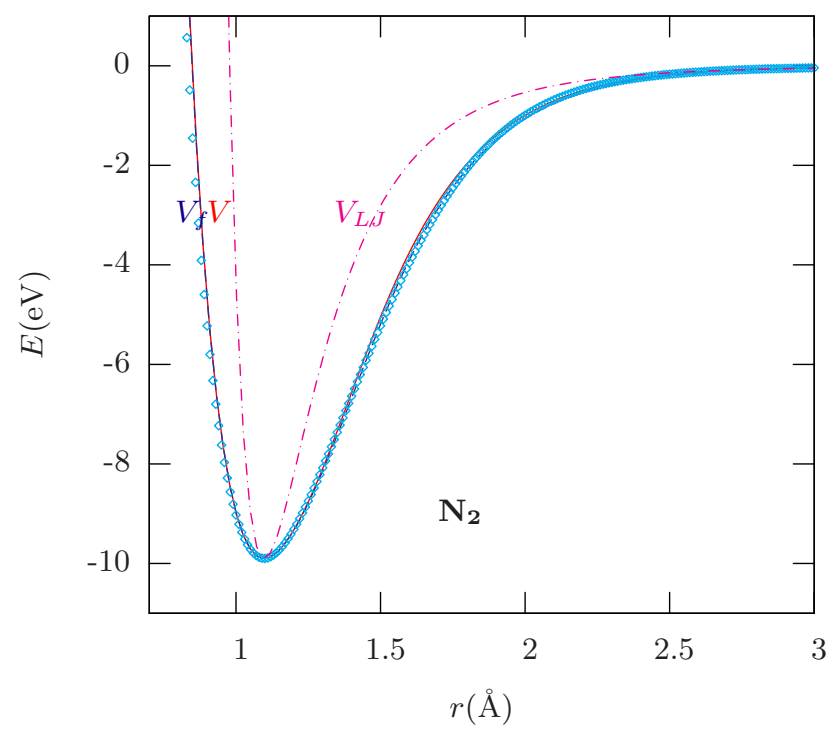

FIG. 2: The hybrid form $V$ with the calculated coefficients ( $a, b, c, \& d$, Table III) (solid, red) fits the RKR spectral points for the ground state of molecular nitrogen (diamonds, cyan) nearly as well as does the hybrid form $V_{f}$ with the fitted coefficients ( $a, b, c, \& d$, Table II) (dashes, dark blue). The Lennard-Jones form $V_{L J}$ (dot-dash, magenta) fits only near the minimum.
TABLE III: Values of the coefficients $a, b, c$, and $d$ obtained by Eqs. 8, 15, 17, 19, \& 20) from the values of $E_{0}, r_{0}, k$, and $C_{6}$ of Table

\begin{tabular}{|c|c|c|c|c|c|}
\hline \hline & $a(\mathrm{eV})$ & $b\left(\AA^{-1}\right)$ & $c\left(\AA^{-1}\right)$ & $d\left(\AA^{12}\right)$ & $\mathrm{f}, \Delta V$ \\
\hline $\mathrm{H}_{2}$ & 45.01 & 2.907 & 2.5663 & 16.7 & $0.68,0.087$ \\
\hline $\mathrm{N}_{2}$ & 4059.02 & 4.435 & 1.1762 & 27.7 & $0.81,0.111$ \\
\hline $\mathrm{O}_{2}$ & 2868.48 & 4.246 & 1.0539 & 40.7 & $0.84,0.068$ \\
\hline $\mathrm{NO}$ & 4040.42 & 4.496 & 1.0946 & 34.0 & $0.81,0.156$ \\
\hline $\mathrm{OH}$ & 491.96 & 3.942 & 1.4478 & 19.8 & $0.81,0.053$ \\
\hline $\mathrm{I}_{2}$ & 16125.67 & 2.832 & 0.4350 & $2.79 \mathrm{e} 5$ & $0.88,0.041$ \\
\hline $\mathrm{Li}_{2}$ & 148.93 & 1.516 & 0.5161 & $4.01 \mathrm{e} 6$ & $0.86,0.028$ \\
\hline $\mathrm{Na}_{2}$ & 278.20 & 1.595 & 0.4275 & $7.33 \mathrm{e} 6$ & $0.81,0.012$ \\
\hline $\mathrm{K}_{2}$ & 359.65 & 1.381 & 0.3273 & $7.30 \mathrm{e} 7$ & $0.85,0.012$ \\
\hline $\mathrm{Ar}_{2}$ & 4994.79 & 2.921 & 0.2959 & $3.12 \mathrm{e} 7$ & $0.70,0.012$ \\
\hline $\mathrm{Kr}_{2}$ & 9610.07 & 2.805 & 0.2759 & $6.23 \mathrm{e} 7$ & $0.90,0.0004$ \\
\hline \hline
\end{tabular}

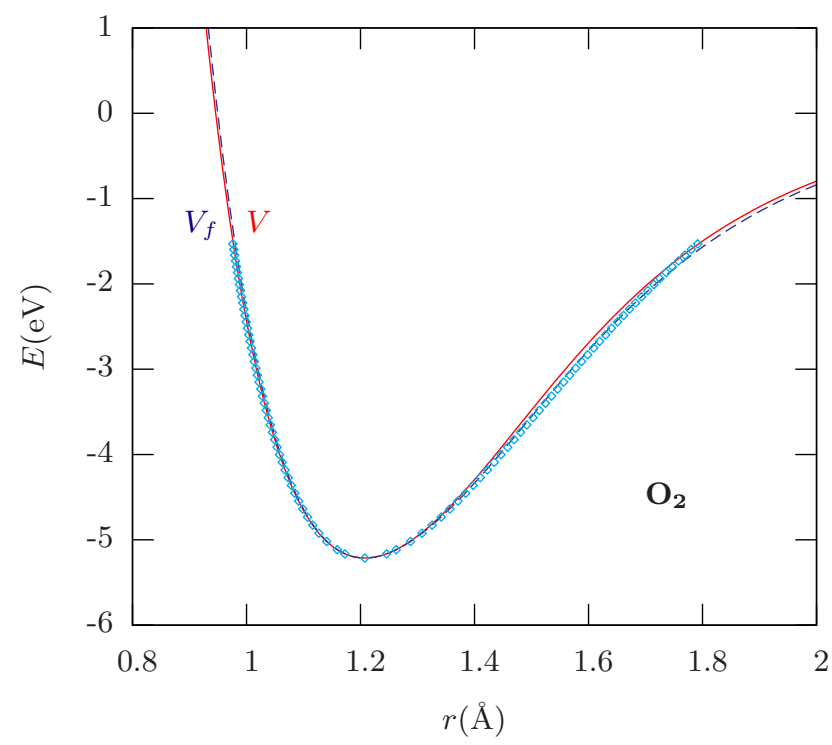

FIG. 3: The hybrid form $V$ with the calculated coefficients ( $a, b, c, \& d$, Table III) (solid, red) fits the RKR spectral points for the ground state of molecular oxygen (diamonds, cyan) nearly as well as does the hybrid form $V_{f}$ with the fitted coefficients ( $a, b, c, \& d$, Table II) (dashes, dark blue).

\section{THREE FORMULAS}

Suppose we use the rule (8) for the fudge-factor $d$ and take the London coefficient $C_{6}$ from Table [. How do we find the parameters $a, b$, and $c$ that make the hybrid potential $V(r)$ have its minimum at $r=r_{0}$ with $V\left(r_{0}\right)=$ $-E_{0}$ and with curvature $V^{\prime \prime}\left(r_{0}\right)=k$ ?

Let us write the hybrid form as the sum

$$
V(r)=v(r)+w(r)
$$

of the $a, b, c$ terms $v(r)$

$$
v(r)=a e^{-b r}(1-c r)
$$




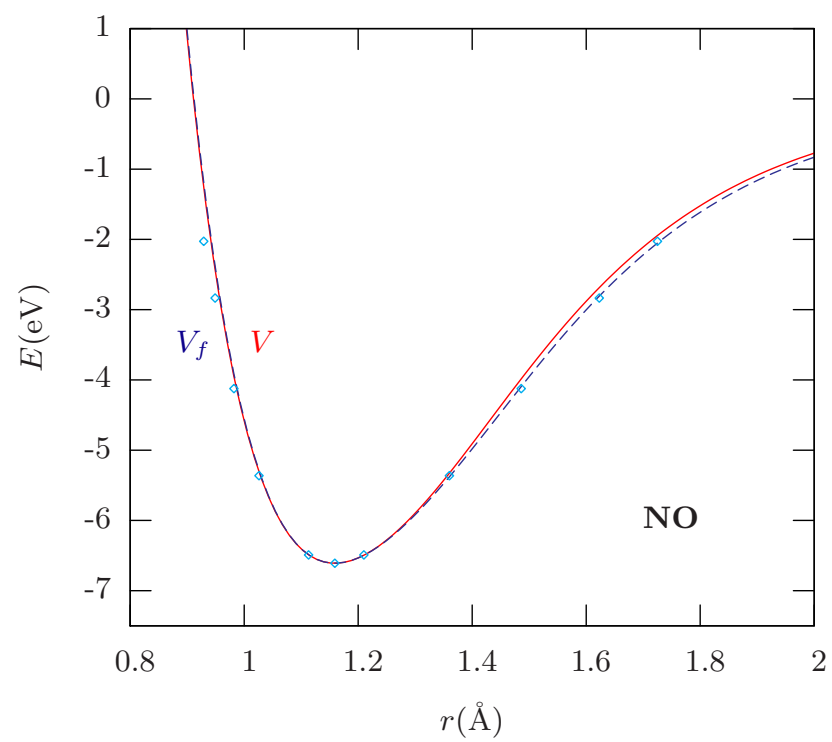

FIG. 4: The hybrid form $V$ with the calculated coefficients ( $a$, $b, c, \& d$, Table III) (solid, red) fits the RKR spectral points for the ground state of nitric oxide (diamonds, cyan) nearly as well as does the hybrid form $V_{f}$ with the fitted coefficients ( $a, b, c, \& d$, Table (dashes, dark blue).

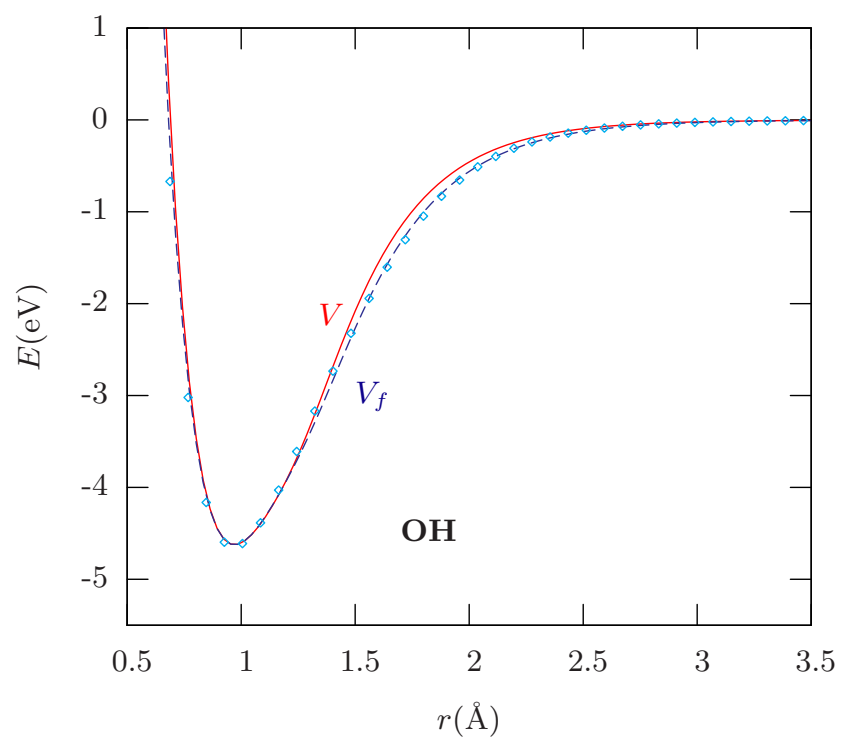

FIG. 5: The hybrid form $V$ with the calculated coefficients ( $a, b, c, \& d$, Table III) (solid, red) fits the RKR spectral points for the ground state of the hydroxyl radical (diamonds, cyan) slightly less well than the hybrid form $V_{f}$ with the fitted coefficients $(a, b, c, \& d$, Table II) (dashes, dark blue).

and the $C_{6}, d$ terms $w(r)$

$$
w(r)=-\frac{C_{6}}{r^{6}+d r^{-6}} .
$$

Since $C_{6}$ and $d$ are given, the function $w(r)$ and its deriva-

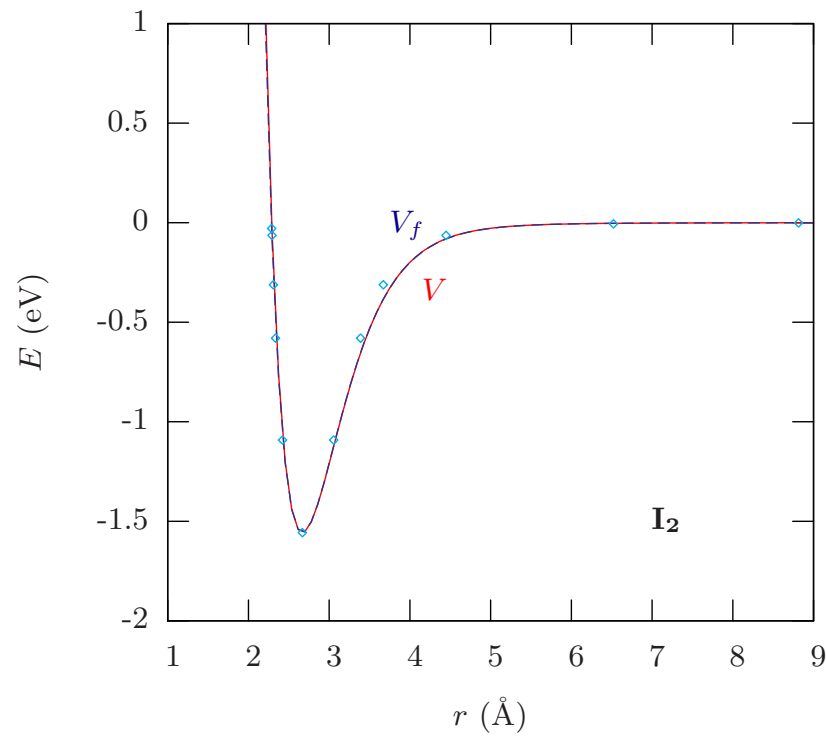

FIG. 6: The hybrid form $V$ with the calculated coefficients ( $a, b, c, \& d$, Table III) (solid, red) fits the RKR spectral points for the ground state of molecular iodine (diamonds, cyan) nearly as well as does the hybrid form $V_{f}$ with the fitted coefficients ( $a, b, c, \& d$, Table (dashes, dark blue).

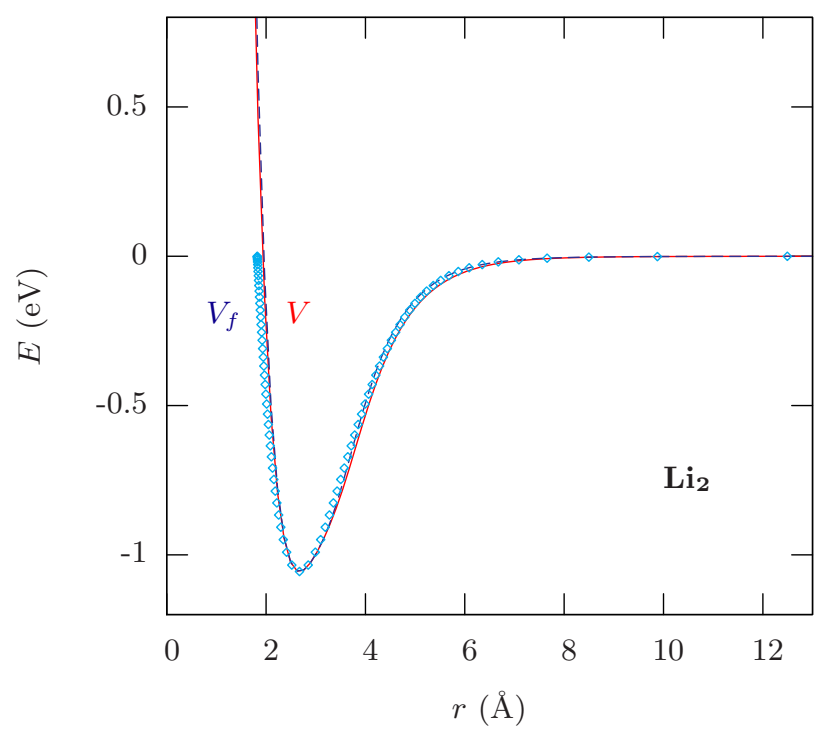

FIG. 7: The hybrid form $V$ with the calculated coefficients ( $a, b, c, \& d$, Table III) (solid, red) fits the RKR spectral points for the ground state of the lithium dimer (diamonds, cyan) nearly as well as does the hybrid form $V_{f}$ with the fitted coefficients ( $a, b, c, \& d$, Table I) (dashes, dark blue).

tives

$$
w^{\prime}(r)=6 C_{6} r^{5} \frac{r^{12}-d}{\left(r^{12}+d\right)^{2}}
$$




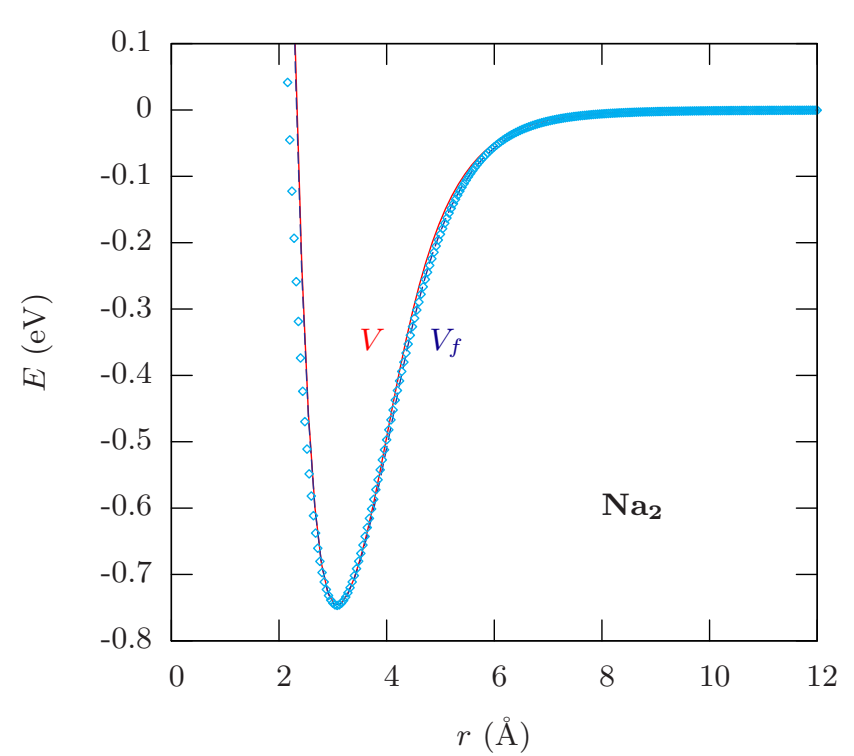

FIG. 8: The hybrid form $V$ with the calculated coefficients ( $a$, $b, c, \& d$, Table III) (solid, red) fits the RKR spectral points for the ground state of the sodium dimer (diamonds, cyan) well, but not quite as well as does the hybrid form $V_{f}$ with the fitted coefficients $(a, b, c, \& d$, Table II) (dashes, dark blue).

and

$$
w^{\prime \prime}(r)=-6 C_{6} r^{4} \frac{\left(7 r^{12}-d\right)\left(r^{12}-5 d\right)}{\left(r^{12}+d\right)^{3}}
$$

are determined. The condition that $V\left(r_{0}\right)=-E_{0}$ then is

$$
a e^{-b r_{0}}\left(1-c r_{0}\right)=-w\left(r_{0}\right)-E_{0}
$$

which implies that $a$ is

$$
a=-\left(w\left(r_{0}\right)+E_{0}\right) e^{b r_{0}} /\left(1-c r_{0}\right) .
$$

The condition that $V^{\prime}\left(r_{0}\right)=0$ is

$$
a e^{-b r_{0}}\left(b+c-b c r_{0}\right)=w^{\prime}\left(r_{0}\right)
$$

which together with (15) gives $b$ as

$$
b=-c /\left(1-c r_{0}\right)-w^{\prime}\left(r_{0}\right) /\left(w\left(r_{0}\right)+E_{0}\right) .
$$

Finally, the condition $V^{\prime \prime}\left(r_{0}\right)=k$ is

$$
a b e^{-b r_{0}}\left(b-b c r_{0}+2 c\right)=k-w^{\prime \prime}\left(r_{0}\right)
$$

which with (15) for $a$ and (17) for $b$ is a quadratic equation for $c$ with roots

$$
c=\left[r_{0} \pm{\sqrt{\frac{\left(w\left(r_{0}\right)+E_{0}\right)^{2}}{w^{\prime}\left(r_{0}\right)^{2}+\left(k-w^{\prime \prime}\left(r_{0}\right)\right)\left(w\left(r_{0}\right)+E_{0}\right)}}}^{-1} .\right.
$$

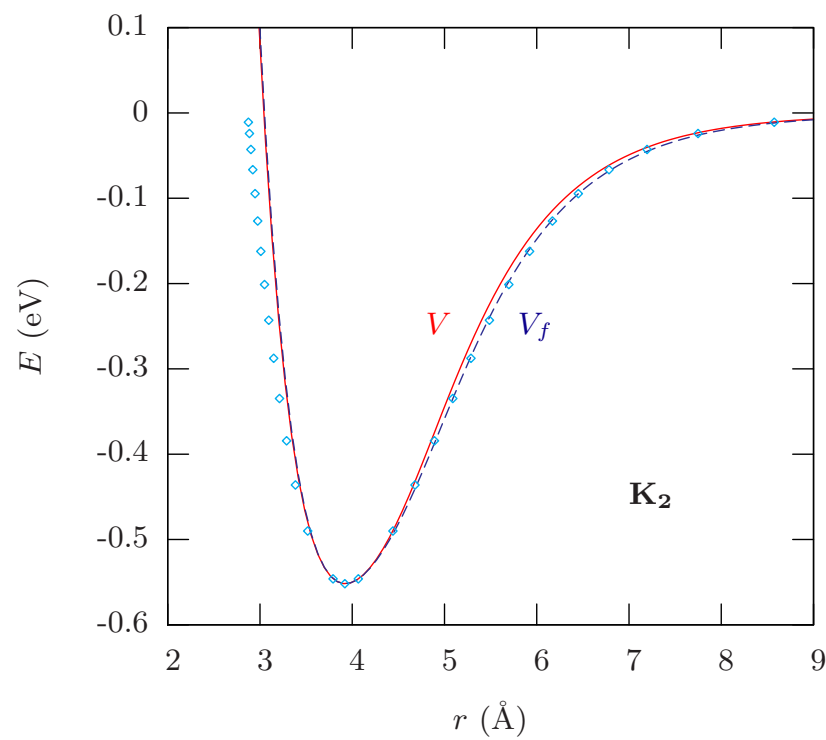

FIG. 9: The hybrid form $V$ with the calculated coefficients ( $a$, $b, c, \& d$, Table III) (solid, red) fits the RKR spectral points for the ground state of the potassium dimer (diamonds, cyan) well, but not quite as well as does the hybrid form $V_{f}$ with the fitted coefficients $(a, b, c, \& d$, Table II) (dashes, dark blue).

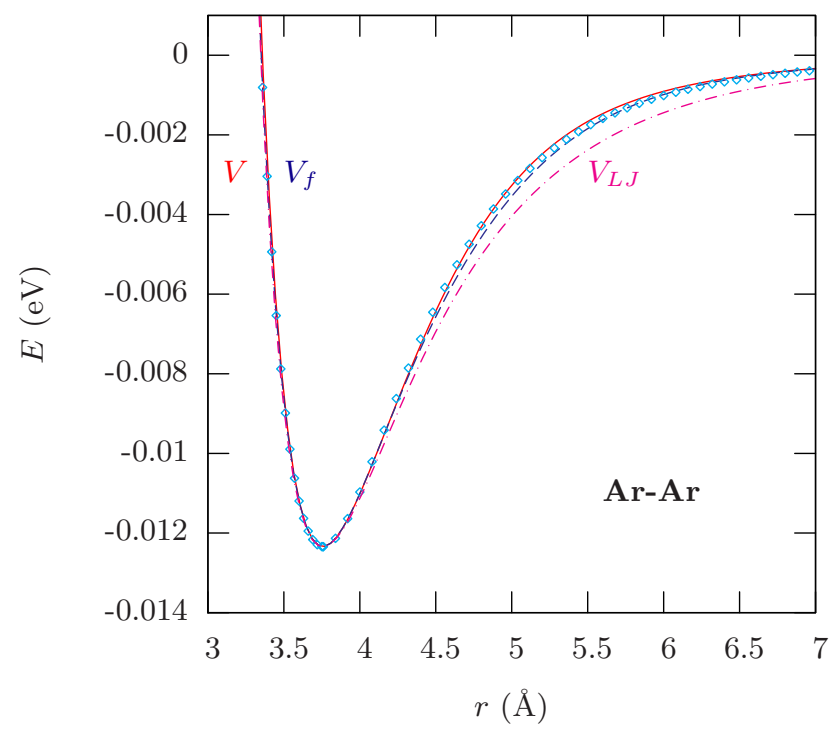

FIG. 10: The hybrid form $V$ with the calculated coefficients ( $a, b, c, \& d$, Table III) (solid, red) fits the RKR spectral points for the ground state of the argon dimer (diamonds, cyan) nearly as well as does the hybrid form $V_{f}$ with the fitted coefficients ( $a, b, c, \& d$, Table II) (dashes, dark blue). The Lennard-Jones form $V_{L J}$ fitted to the minimum (dot-dash, magenta) is too low for $r>4 \AA$. 


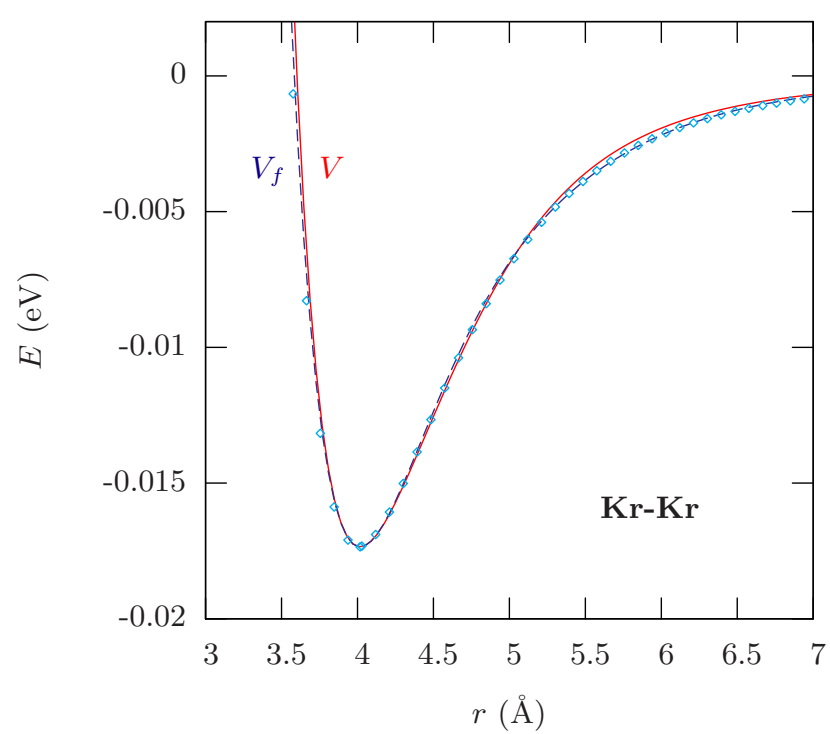

FIG. 11: The hybrid form $V$ with the calculated coefficients $(a, b, c, \& d$, Table III) (solid, red) fits the RKR spectral points for the ground state of the krypton dimer (diamonds, cyan) nearly as well as does the hybrid form $V_{f}$ with the fitted coefficients $(a, b, c, \& d$, Table (dashes, dark blue).

The minus sign implies

$$
c>1 / r_{0} \Longleftrightarrow 1-c r_{0}<0
$$

while the plus sign implies

$$
c<1 / r_{0} \Longleftrightarrow 1-c r_{0}>0 .
$$

A covalently bonded pair will have $v\left(r_{0}\right)<0$, which implies $1-c r_{0}<0$ and so $c>1 / r_{0}$, which entails the minus sign. One may choose the minus sign for the 11 pairs of neutral atoms considered in this paper. A further discussion of the choice of sign appears in the Appendix.

If one uses the rule of thumb (8) for $d$ and the values of $r_{0}, E_{0}, k, \& C_{6}$ from Table प, then one may find $c$ from condition (19) with a choice of sign, and then $b$ from (17), and then $a$ from (15). The resulting values of $a, b, c, \&$ $d$ for the 11 pairs of neutral atoms $\mathrm{H}_{2}, \mathrm{~N}_{2}, \mathrm{O}_{2}, \mathrm{NO}, \mathrm{OH}$, $\mathrm{I}_{2}, \mathrm{Li}_{2}, \mathrm{Na}_{2}, \mathrm{~K}_{2}, \mathrm{Ar}-\mathrm{Ar}, \& \mathrm{Kr}-\mathrm{Kr}$ are listed in Table for the case of a minus sign in Eq. (19); also shown are the rms errors $\Delta V(r)$ for $r \geq f r_{0}$ for the $f$ 's of Table [I] Figures 111 show the hybrid form $V$ with these values of $a, b, c, \& d$ and with the $C_{6}$ 's of Table $\square$ as solid red curves. They closely trace the empirical potentials (diamonds, cyan) and the fitted hybrid forms $V_{f}$ (dashes, dark blue).

\section{CONCLUSIONS}

By using Eqs. (8) 15, 17, 19, \& 20), one may build an accurate hybrid potential (3) for the ground state of a pair of neutral atoms from their internuclear separation, the depth and curvature of their potential at its minimum, and from their van der Waals coefficient $C_{6}$. The hybrid potential therefore is applicable to pairs of neutral atoms for which no empirical potential is available. Given the differences between it and the Lennard-Jones, harmonic, Morse, Varnshi, and Hulburt-Hirschfelder potentials, it would be worthwhile to examine the consequences of these differences in Monte Carlo searches for low-energy states of biomolecules and in numerical simulations of phase transitions and reactions far from equilibrium.

\section{Acknowledgments}

Thanks to G. Groenenboom for sending me a copy of the potential of 28] and to S. Atlas, S. T. P. Boyd, H.-B. Broeker, D. Cromer, K. Dill, M. Fleharty, S. C. Foster, R. J. Le Roy, W. J. Meath, V. A. Parsegian, R. Pastor, R. Podgornik, E. Riley, B. Rivers, D. Sergatskov, J. Thomas, S. Valone and W. Zemke for advice.

\section{APPENDIX: THE CHOICE OF SIGN}

The choice of the minus sign in Eq. (19) implies that the fudge factor $d$ must exceed a certain lower limit. The parameter $a$ is the value of the potential $V(r)$ at $r=0$ and so must be positive. The minus-sign inequalities (20) imply that $1-c r_{0}<0$. Thus Eq. (15) will give a positive value for $a$ only if

$$
w\left(r_{0}\right)+E_{0}>0
$$

which by (11) implies the lower limit

$$
d>\frac{C_{6} r_{0}^{6}}{E_{0}}-r_{0}^{12}
$$

on the fudge factor $d$. The values of $d$ listed in Tables \& III satisfy this constraint.

The choice of the plus sign in Eq.(19) implies two upper limits on the fudge factor $d$. The plus-sign inequalities (21) imply that $1-c r_{0}>0$. Thus Eq.(15) will give a positive value for $a$ only if

$$
w\left(r_{0}\right)+E_{0}<0
$$

which by (11) implies the upper limit

$$
d<\frac{C_{6} r_{0}^{6}}{E_{0}}-r_{0}^{12}
$$

on the fudge factor $d$. The right-hand-side of this inequality is negative for the pairs $\mathrm{N}_{2}, \mathrm{O}_{2}, \mathrm{NO}, \& \mathrm{I}_{2}$ and less than unity for the pairs $\mathrm{H}_{2} \& \mathrm{OH}$, as may be seen from the values of $C_{6}, r_{0}, \& E_{0}$ listed in Table I. Thus the minus sign is required for the pairs $\mathrm{N}_{2}, \mathrm{O}_{2}, \mathrm{NO}, \& \mathrm{I}_{2}$ and strongly indicated for the pairs $\mathrm{H}_{2} \& \mathrm{OH}$. 
TABLE IV: The values of the coefficients $a, b, c, \& d$ that best fit the hybrid potential $V(r)$ (Eq. (3)) to RKR data for $\mathrm{Li}_{2}$, $\mathrm{Na}_{2}, \& \mathrm{~K}_{2}$ and to empirical potentials for $\mathrm{Ar}-\mathrm{Ar} \& \mathrm{Kr}-\mathrm{Kr}$ while respecting Eqs. (15, 17, \& 19) with a plus sign in (19). $\Delta V(r)$ is the rms error in $V$ for $r \geq f r_{0}$.

\begin{tabular}{|l|c|c|c|c|c|}
\hline \hline & $a(\mathrm{eV})$ & $b\left(\AA^{-1}\right)$ & $c\left(\AA^{-1}\right)$ & $d\left(\AA^{12}\right)$ & $f, \Delta V(\mathrm{eV})$ \\
\hline $\mathrm{Li}_{2}$ & 1136.21 & 1.8218 & 0.3225 & 869. & $0.77,0.037$ \\
\hline $\mathrm{Na}_{2}$ & 648.22 & 1.5692 & 0.3034 & 2692. & $0.90,0.004$ \\
\hline $\mathrm{K}_{2}$ & 500.31 & 1.2673 & 0.2486 & 33770. & $0.70,0.012$ \\
\hline $\mathrm{Ar}_{2}$ & 1754.01 & 2.7054 & 0.2620 & $1.57 \mathrm{e} 5$ & $0.70,0.001$ \\
\hline $\mathrm{Kr}_{2}$ & 2725.43 & 2.5622 & 0.2485 & $1.0 \mathrm{e} 6$ & $0.90,0.0003$ \\
\hline \hline
\end{tabular}

The second upper limit on $d$ arises because both $b$ and $c$ must be positive $(b>0$ because $v(r)$ must vanish as $r \rightarrow \infty$, and $c>0$ because $c<0$ implies $v(r)>0$ ). Thus if the positive sign is chosen in the formula (19) for $c$, then $1-c r_{0}>0$, and also $w\left(r_{0}\right)+E_{0}<0$ by A.3. and so Eq.(17) will give a positive value for $b$ only if $w^{\prime}\left(r_{0}\right)>0$, which by (12) implies the upper limit

$$
d<r_{0}^{12}
$$

The curves displayed in Figs. 1111 all correspond to the choice of a minus sign in Eq.(19). But for the five pairs $\mathrm{Li}_{2}, \mathrm{Na}_{2}, \mathrm{~K}_{2}, \mathrm{Ar}-\mathrm{Ar}, \& \mathrm{Kr}-\mathrm{Kr}$, one also may get good fits to the empirical data by using the plus sign (21). The resulting values of $a, b, c, \& d$ appear in Table IV. The $d$ 's obey A.4 \& A.5.
[1] J. E. Lennard-Jones, Proc. Phys. Soc. 43, 461 (1931).

[2] K. Cahill and V. A. Parsegian, J. Chem. Phys. 121, 10839 (2004), arxiv.org/q-bio.BM/0410018.

[3] P. Ren and J. W. J. W. Ponder, J. Phys. Chem. B 107, 5933 (2003).

[4] D. A. Pearlman, D. A. Case, J. W. Caldwell, W. S. Ross, T. E. Cheatham III, S. DeBolt, D. Ferguson, G. Seibel, and P. Kollman, Comp. Phys. Commun. 91, 1 (1995).

[5] K. Cahill and V. A. Parsegian (2003), q-bio.BM/0312005

[6] P. M. Morse, Phys. Rev. 34, 57 (1929).

[7] Y. P. Varnshi, Rev. Mod. Phys. 29, 664 (1957).

[8] H. M. Hulbert and J. O. Hirschfelder, J. Chem. Phys. 35, 1901(L) (1961).

[9] D. Steele, E. R. Lippincott, and J. T. Vanderslice, Rev. Mod. Phys. 34, 239 (1962).

[10] P. H. Krupenie, J. Phys. Chem. Ref. Data 1, 423 (1972).

[11] J. Mitroy and M. W. J. Bromley, Phys. Rev. A 71, 032709 (2005).

[12] G. D. Zeiss and W. J. Meath, Mol. Phys. 33, 1155 (1977).

[13] A. J. C. Varandas and A. I. Voronin, Chem. Phys. 194(1), 91 (1995).

[14] X. Chu and A. Dalgarno, J. Chem. Phys. 121(9), 4083 (2004).

[15] R. A. Aziz, J. Chem. Phys. 99, 4518 (1993).

[16] A. K. Dham, A. R. Allnatt, W. J. Meath, and R. A. Aziz,
Mol. Phys. 67, 1291 (1989).

[17] R. J. Le Roy, Y. Yiye Huang, and C. Jary, J. Chem. Phys. 125(16), 164309 (2006).

[18] J. A. Coxon and T. C. Melville, J. Mol. Spectrosc. 235(2), 235 (2006).

[19] C. Samuelis, E. Tiesinga, T. Laue, M. Elbs, H. Knöckel, and E. Tiemann, Phys. Rev. A 63(1), 012710 (2000).

[20] A. Derevianko, W. R. Johnson, M. S. Safronova, and J. F. Babb, Phys. Rev. Lett. 82(18), 3589 (1999).

[21] C. Amiot, J. Vergès, and C. E. Fellows, J. Chem. Phys. 103(9), 3350 (1995).

[22] C. Amiot, J. Mol. Spectrosc. 147, 370 (1991).

[23] S. Weissman, J. T. Vanderslice, and R. Battino, J. Chem. Phys. 39, 2226 (1963).

[24] A. Lofthus and P. H. Krupenie, J. Phys. Chem. Ref. Data 6, 113 (1977).

[25] R. Rydberg, Z. Phys. 73, 376 (1931).

[26] O. Klein, Z. Phys. 76, 226 (1932).

[27] A. L. G. Rees, Proc. Phys. Soc. (London) 59, 998 (1947).

[28] M. P. J. van der Loo and G. C. Groenenboom, J. Chem. Phys. 126, 114314 (2007).

[29] R. Côté, A. Dalgarno, H. Wang, and W. C. Stwalley, Phys. Rev. A 57, R4118 (1998). 